\title{
SECOND ORDER ASYMPTOTIC VARIANCE OF THE BAYES ESTIMATOR OF A TRUNCATION PARAMETER FOR A ONE-SIDED TRUNCATED EXPONENTIAL FAMILY OF DISTRIBUTIONS
}

\author{
Masafumi Akahira*
}

\begin{abstract}
For a one-sided truncated exponential family of distributions with a truncation parameter $\gamma$ and a natural parameter $\theta$ as a nuisance parameter, the stochastic expansions of the Bayes estimator $\hat{\gamma}_{B, \theta}$ when $\theta$ is known and the Bayes estimator $\hat{\gamma}_{B, \hat{\theta}_{M L}}$ plugging the maximum likelihood estimator (MLE) $\hat{\theta}_{M L}$ in $\theta$ of $\hat{\gamma}_{B, \theta}$ when $\theta$ is unknown are derived. The second order asymptotic loss of $\hat{\gamma}_{B, \hat{\theta}_{M L}}$ relative to $\hat{\gamma}_{B, \theta}$ is also obtained through their asymptotic variances. Further, it is shown that $\hat{\gamma}_{B, \theta}$ and $\hat{\gamma}_{B, \hat{\theta}_{M L}}$ are second order asymptotically equivalent to the bias-adjusted MLEs $\hat{\gamma}_{M L^{*}, \theta}$ and $\hat{\gamma}_{M L^{*}}$ when $\theta$ is known and when $\theta$ is unknown, respectively. Some examples are also given.
\end{abstract}

Key words and phrases: Asymptotic variance, Bayes estimator, maximum likelihood estimator, second order asymptotic loss, stochastic expansion, truncation parameter.

\section{Introduction}

In order to compare asymptotically efficient estimators, we may calculate their asymptotic variances up to the higher order. In such cases, the concept of asymptotic deficiency discussed by Hodges and Lehmann (1970) plays an important part. Indeed, for two estimators $\hat{\theta}_{n}^{(1)}$ and $\hat{\theta}_{n}^{(2)}$ of $\theta$ based on a sample of size $n$, let $d_{n}$ be an additional size of sample needed such that $\hat{\theta}_{n}^{(2)}$ is asymptotically equivalent to $\hat{\theta}_{n}^{(1)}$ in some sense. If $\lim _{n \rightarrow \infty} d_{n}$ exists, then it is called the asymptotic deficiency of $\hat{\theta}_{n}^{(2)}$ relative to $\hat{\theta}_{n}^{(1)}$, which is useful in comparing asymptotically efficient estimators up to the higher order (see Akahira (1986) under suitable regularity conditions).

In the presence of nuisance parameters, the asymptotic deficiencies of the maximum likelihood estimator (MLE) based on the pooled sample were discussed by Akahira and Takeuchi (1982). Ferguson (1992) also considered the inference on an interest parameter in the presence of nuisance parameters in terms of the conditional maximum likelihood estimator developed by Cox and Reid (1987).

For a truncated exponential family of distributions with a natural parameter $\theta$ and a truncation parameter $\gamma$ as a nuisance parameter, Bar-Lev (1984) showed that the MLE $\hat{\theta}_{M L}^{\gamma}$ of $\theta$ for known $\gamma$, and the MLE $\hat{\theta}_{M L}$ and the maximum conditional likelihood estimator (MCLE) $\hat{\theta}_{M C L}$ of $\theta$ for unknown $\gamma$ have the same asymptotic normality, and Akahira (2016) obtained the second order asymptotic

Received January 13, 2016. Revised May 20, 2016. Accepted July 8, 2016.

*Institute of Mathematics, University of Tsukuba, Ibaraki 305-8571, Japan. 
losses of the bias-adjusted MLE $\hat{\theta}_{M L}^{*}$ and $\hat{\theta}_{M C L}$ relative to $\hat{\theta}_{M L}^{\gamma}$ through their asymptotic variances which correspond to the asymptotic deficiencies calculated from their stochastic expansion. Note that the truncated exponential family involves the Pareto distribution (see also Arnold (2015)). On the other hand, Akahira and Ohyauchi (2016a) considered an estimation problem on a truncation parameter $\gamma$ when a natural parameter $\theta$ is a nuisance one, and gave the stochastic expansions of bias-adjusted MLEs $\hat{\gamma}_{M L^{*}}^{\theta}$ and $\hat{\gamma}_{M L^{*}}$ of $\gamma$ for known $\theta$ and unknown $\theta$, respectively. The second order asymptotic loss of $\hat{\gamma}_{M L^{*}}$ relative $\hat{\gamma}_{M L^{*}}^{\theta}$ was also obtained through their asymptotic variances.

In this paper, as a continuated work of Akahira and Ohyauchi (2016a), the estimation problem on $\gamma$ is considered from the Bayesian viewpoint. In Section 2, we give formulation and assumptions. In Section 3 , when $\theta$ is known, the stochastic expansion of the Bayes estimator $\hat{\gamma}_{B, \theta}$ of $\gamma$ is derived, and the asymptotic mean and asymptotic variance of $\hat{\gamma}_{B, \theta}$ are given. In Section 4 , when $\theta$ is unknown, the stochastic expansion of the Bayes estimator $\hat{\gamma}_{B, \hat{\theta}_{M L}}$ plugging the MLE $\hat{\theta}_{M L}$ in $\theta$ of $\hat{\gamma}_{B, \theta}$ is derived, and the asymptotic mean and asymptotic variance of $\hat{\gamma}_{B, \hat{\theta}_{M L}}$ are given. In Section 5, some examples including a truncated Beta distribution and a truncated Erlang distribution are given. In Appendix, the proofs of Theorems 1 and 2 are given. Here, the asymptotic mean and asymptotic variance are based on the definition of asymptotic expectation (see Akahira and Takeuchi (1987)).

\section{Formulation and assumptions}

In a similar way to Bar-Lev (1984), Akahira (2016) and Akahira and Ohyauchi (2016a), we suppose that $X_{1}, X_{2}, \ldots, X_{n}, \ldots$ is a sequence of independent and identically distributed (i.i.d.) random variables according to $P_{\theta, \gamma}$, having a density

$$
f(x ; \theta, \gamma)= \begin{cases}\frac{a(x) e^{\theta u(x)}}{b(\theta, \gamma)} & \text { for } c<\gamma \leq x<d, \\ 0 & \text { otherwise }\end{cases}
$$

with respect to the Lebesgue measure, where $-\infty \leq c<d \leq \infty, a(\cdot)$ is nonnegative and continuous almost surely, and $u(\cdot)$ is absolutely continuous with $d u(x) / d x \not \equiv 0$ over the interval $(\gamma, d)$. Let

$$
\Theta(\gamma):=\left\{\theta \mid 0<b(\theta, \gamma):=\int_{\gamma}^{d} a(x) e^{\theta u(x)} d x<\infty\right\}
$$

for $\gamma \in(c, d)$. Then it is shown that for any $\gamma_{1}, \gamma_{2} \in(c, d)$ with $\gamma_{1}<\gamma_{2}$, $\Theta\left(\gamma_{1}\right) \subset \Theta\left(\gamma_{2}\right)$. Assume that for any $\gamma \in(c, d), \Theta \equiv \Theta(\gamma)$ is a non-empty open interval. A family $\mathcal{P}:=\left\{P_{\theta, \gamma} \mid \theta \in \Theta, \gamma \in(c, d)\right\}$ of distributions $P_{\theta, \gamma}$ having a density (2.1) with a truncation parameter $\gamma$ and a natural parameter $\theta$ is called a one-sided truncated exponential family of distributions.

Let $\pi(\gamma)$ be a prior density with respect to the Lebesgue measure over the open interval $(c, d)$, and $L(\hat{\gamma}, \gamma)$ the quadratic loss $(\hat{\gamma}-\gamma)^{2}$ of any estimator 
$\hat{\gamma}=\hat{\gamma}(\boldsymbol{X})$ based on $\boldsymbol{X}:=\left(X_{1}, \ldots, X_{n}\right)$. Suppose that $\theta$ is known. Then it is easily seen that the Bayes estimator of $\gamma$ w.r.t. $L$ and $\pi$ is given by

$$
\hat{\gamma}_{B, \theta}(\boldsymbol{X}):=\int_{c}^{X_{(1)}} \frac{t \pi(t)}{b^{n}(\theta, t)} d t / \int_{c}^{X_{(1)}} \frac{\pi(t)}{b^{n}(\theta, t)} d t,
$$

where $X_{(1)}:=\min _{1 \leq i \leq n} X_{i}$. In what follows, we always assume that $a(\cdot)$ and $u(\cdot)$ are functions of class $\mathbb{C}^{3}$ and $\pi(\cdot)$ is a function of class $\mathbb{C}^{2}$ on the interval $(c, d)$.

\section{The Bayes estimator $\hat{\gamma}_{B, \theta}$ of $\gamma$ when $\theta$ is known}

Letting $v=n(t-\gamma)$, we have from $(2.3)$

$$
\hat{\gamma}_{B, \theta}(\boldsymbol{X})=\gamma+\frac{1}{n}\left(\int_{\tau_{n}}^{T_{(1)}} \frac{v \pi\left(\gamma+\frac{v}{n}\right)}{b^{n}\left(\theta, \gamma+\frac{v}{n}\right)} d v / \int_{\tau_{n}}^{T_{(1)}} \frac{\pi\left(\gamma+\frac{v}{n}\right)}{b^{n}\left(\theta, \gamma+\frac{v}{n}\right)} d v\right)
$$

where $\tau_{n}:=n(c-\gamma)$ and $T_{(1)}:=n\left(X_{(1)}-\gamma\right)$. Let

$$
\begin{array}{ll}
b_{(j)}(\theta, \gamma):=\frac{\partial^{j}}{\partial \gamma^{j}} \log b(\theta, \gamma) & (j=1,2, \ldots), \\
\pi_{(j)}(\gamma):=\frac{\partial^{j}}{\partial \gamma^{j}} \log \pi(\gamma) & (j=1,2, \ldots) .
\end{array}
$$

It is noted from (2.2) that

$$
k(\theta, \gamma):=\frac{a(\gamma) e^{\theta u(\gamma)}}{b(\theta, \gamma)}=-b_{(1)}(\theta, \gamma) .
$$

Then we have the following.

THEOREM 1. For the one-sided truncated exponential family $\mathcal{P}$ of distributions having a density (2.1) with a truncation parameter $\gamma$ and a natural parameter $\theta$, let $\hat{\gamma}_{B, \theta}$ be the Bayes estimator (2.3) of $\gamma$ w.r.t. the loss $L$ and the prior density $\pi$, when $\theta$ is known. Then the stochastic expansion of $T_{B, \theta}:=n\left(\hat{\gamma}_{B, \theta}-\gamma\right)$ is given by

$$
\begin{aligned}
T_{B, \theta}= & T_{(1)}-\frac{1}{k}+\frac{1}{k n}\left(\frac{\partial}{\partial \gamma} \log k\right) T_{(1)} \\
& -\frac{1}{k^{2} n}\left\{2\left(\frac{\partial}{\partial \gamma} \log k\right)-\pi_{(1)}\right\}+O_{p}\left(\frac{1}{n^{2}}\right),
\end{aligned}
$$

and the asymptotic mean and asymptotic variance of $k T_{B, \theta}$ are given by

$$
\begin{aligned}
& E_{\gamma}\left(k T_{B, \theta}\right)=-\frac{1}{k n}\left\{2\left(\frac{\partial}{\partial \gamma} \log k\right)-\pi_{(1)}\right\}+O\left(\frac{1}{n^{2}}\right), \\
& V_{\gamma}\left(k T_{B, \theta}\right)=1-\frac{2}{k n}\left(\frac{\partial}{\partial \gamma} \log k\right)+O\left(\frac{1}{n^{2}}\right),
\end{aligned}
$$


respectively, where $k=k(\theta, \gamma)$ and $\pi_{(1)}=\pi_{(1)}(\gamma)$ (the asymptotic variance of $k T_{B, \theta}$ is free of the prior density $\left.\pi\right)$.

Remark 1. We see that the part except the term

$$
-\frac{1}{k^{2} n}\left\{2\left(\frac{\partial}{\partial \gamma} \log k\right)-\pi_{(1)}\right\}
$$

in the right-hand side of (3.5) coincides with the stochastic expansion of $T_{(1)}^{*}:=$ $n\left(X_{(1)}^{*}-\gamma\right)$ up to the order $n^{-1}$, where $X_{(1)}^{*}:=X_{(1)}-\left(\hat{k}_{\theta} n\right)^{-1}$ is the bias-adjusted MLE $\hat{\gamma}_{M L^{*}}^{\theta}$, with $\hat{k}_{\theta}=k\left(\theta, X_{(1)}\right)$ (see Akahira and Ohyauchi (2016a)). Then, we have

$$
k\left(T_{B, \theta}-T_{(1)}^{*}\right)=-\frac{1}{k n}\left\{2\left(\frac{\partial}{\partial \gamma} \log k\right)-\pi_{(1)}\right\}+O_{p}\left(\frac{1}{n^{2}}\right),
$$

which affects only the difference between their asymptotic means. That is, $\hat{\gamma}_{B, \theta}$ is second order asymptotically equivalent to the bias-adjusted MLE $\hat{\gamma}_{M L^{*}}^{\theta}=X_{(1)}^{*}$, hence

$$
V_{\gamma}\left(k T_{B, \theta}\right)-V_{\gamma}\left(k T_{(1)}^{*}\right)=O\left(\frac{1}{n^{2}}\right) .
$$

\section{The Bayes estimator $\hat{\gamma}_{B, \hat{\theta}_{M L}}$ of $\gamma$ when $\theta$ is unknown}

For any $\gamma \in(c, d), \log b(\theta, \gamma)$ is strictly convex and infinitely differentiable in $\theta \in \Theta$ and

$$
\lambda_{j}(\theta, \gamma):=\frac{\partial^{j}}{\partial \theta^{j}} \log b(\theta, \gamma)
$$

is the $j$-th cumulant corresponding to (2.1) for $j=1,2, \ldots$ For given $\boldsymbol{x}$ satisfying $\gamma \leq x_{(1)}$ and $x_{(n)}<d$, the likelihood function of $\gamma$ and $\theta$ is given by

$$
L(\gamma, \theta ; \boldsymbol{x})=\frac{1}{b^{n}(\theta, \gamma)}\left\{\prod_{i=1}^{n} a\left(x_{i}\right)\right\} \exp \left\{\theta \sum_{i=1}^{n} u\left(x_{i}\right)\right\},
$$

where $\boldsymbol{x}=\left(x_{1}, \ldots, x_{n}\right)$. Let $\hat{\gamma}_{M L}$ and $\hat{\theta}_{M L}$ be the MLEs of $\gamma$ and $\theta$, respectively. From (4.2) it is seen that $\hat{\gamma}_{M L}=X_{(1)}$ and $L\left(X_{(1)}, \hat{\theta}_{M L} ; \boldsymbol{X}\right)=$ $\sup _{\theta \in \Theta} L\left(X_{(1)}, \theta ; \boldsymbol{X}\right)$, hence $\hat{\theta}_{M L}$ satisfies the likelihood equation

$$
\frac{1}{n} \sum_{i=1}^{n} u\left(X_{i}\right)-\lambda_{1}\left(\hat{\theta}_{M L}, X_{(1)}\right)=0 .
$$

Let $\lambda_{2}=\lambda_{2}(\theta, \gamma)$ and $\hat{U}=\sqrt{\lambda_{2} n}\left(\hat{\theta}_{M L}-\theta\right)$. When $\theta$ is unknown, using the MLE $\hat{\theta}_{M L}$ of $\theta$ we consider the Bayes estimator plugging $\hat{\theta}_{M L}$ in $\theta$ of $\hat{\gamma}_{B, \theta}$, i.e.,

$$
\hat{\gamma}_{B, \hat{\theta}_{M L}}(\boldsymbol{X}):=\int_{c}^{X_{(1)}} \frac{t \pi(t)}{b^{n}\left(\hat{\theta}_{M L}, t\right)} d t / \int_{c}^{X_{(1)}} \frac{\pi(t)}{b^{n}\left(\hat{\theta}_{M L}, t\right)} d t .
$$


Then we have the following.

THEOREM 2. For the one-sided the truncated exponential family $\mathcal{P}$ of distributions having a density (2.1) with a truncation parameter $\gamma$ and a natural parameter $\theta$, let $\hat{\gamma}_{B, \hat{\theta}_{M L}}$ be the Bayes estimator (4.4) plugging $\hat{\theta}_{M L}$ in $\theta$ of $\hat{\gamma}_{B, \theta}$ when $\theta$ is unknown. Then the stochastic expansion of $T_{B, \hat{\theta}_{M L}}:=n\left(\hat{\gamma}_{B, \hat{\theta}_{M L}}-\gamma\right)$ is given by

$$
\begin{aligned}
T_{B, \hat{\theta}_{M L}}= & T_{(1)}-\frac{1}{k}+\frac{1}{k^{2} \sqrt{\lambda_{2} n}}\left(\frac{\partial k}{\partial \theta}\right)\left\{\hat{U}+\frac{1}{\sqrt{\lambda_{2} n}}\left(\frac{1}{k}\left(\frac{\partial \lambda_{1}}{\partial \gamma}\right)+\frac{\lambda_{3}}{2 \lambda_{2}}\right)\right\} \\
& +\frac{1}{k n}\left(\frac{\partial}{\partial \gamma} \log k\right) T_{(1)}+\frac{1}{2 k^{2} \lambda_{2} n}\left\{\frac{\partial^{2} k}{\partial \theta^{2}}-\frac{2}{k}\left(\frac{\partial k}{\partial \theta}\right)^{2}\right\}\left(\hat{U}^{2}-1\right) \\
& +\frac{B}{k n}+O_{p}\left(\frac{1}{n \sqrt{n}}\right),
\end{aligned}
$$

where

$$
B:=-\frac{1}{2 \lambda_{2}}\left\{\frac{\lambda_{3}}{k \lambda_{2}}\left(\frac{\partial k}{\partial \theta}\right)-\frac{1}{k}\left(\frac{\partial^{2} k}{\partial \theta^{2}}\right)\right\}-\frac{1}{k}\left\{2\left(\frac{\partial}{\partial \gamma} \log k\right)-\pi_{(1)}\right\},
$$

$k=k(\theta, \gamma), \lambda_{j}=\lambda_{j}(\theta, \gamma)(j=1,2,3)$, and the asymptotic mean and asymptotic variance of $k T_{B, \hat{\theta}_{M L}}$ are given by

$$
\begin{aligned}
& E_{\theta, \gamma}\left[k T_{B, \hat{\theta}_{M L}}\right]=\frac{B}{n}+O\left(\frac{1}{n \sqrt{n}}\right), \\
& V_{\theta, \gamma}\left(k T_{B, \hat{\theta}_{M L}}\right)=1-\frac{2}{k n}\left(\frac{\partial}{\partial \gamma} \log k\right)+\frac{1}{\lambda_{2} n}\left\{u(\gamma)-\lambda_{1}\right\}^{2}+O\left(\frac{1}{n \sqrt{n}}\right),
\end{aligned}
$$

respectively (the asymptotic variance of $k T_{B, \hat{\theta}_{M L}}$ is free of the prior density $\pi$ ).

Remark 2. In the stochastic expansion (4.5), the terms involving $\hat{U}$ yield from the use of the MLE $\hat{\theta}_{M L}$ in $\theta$ of $\hat{\gamma}_{B, \theta}$. It is also seen from (4.6) and (4.7) that the terms depending on $\lambda_{2}$ and $\lambda_{3}$ in the asymptotic mean and asymptotic variance come from using $\hat{\theta}_{M L}$.

Remark 3. We see that the part except the term $B /(k n)$ in the right-hand side of (4.5) coincides with the stochastic expansion of $T_{(1)}^{* *}:=n\left(X_{(1)}^{* *}-\gamma\right)$ up to the order $n^{-1}$, where

$$
\begin{aligned}
X_{(1)}^{* *}:= & X_{(1)}-\frac{1}{\hat{k} n}+\frac{1}{\hat{k}^{2} \hat{\lambda}_{2} n^{2}}\left(\frac{\partial \hat{k}}{\partial \theta}\right)\left\{\frac{1}{\hat{k}}\left(\frac{\partial \hat{\lambda}_{1}}{\partial \gamma}\right)+\frac{\hat{\lambda}_{3}}{2 \hat{\lambda}_{2}}\right\} \\
& -\frac{1}{2 \hat{k}^{2} \hat{\lambda}_{2} n^{2}}\left\{\frac{\partial^{2} \hat{k}}{\partial \theta^{2}}-\frac{2}{\hat{k}}\left(\frac{\partial \hat{k}}{\partial \theta}\right)^{2}\right\}
\end{aligned}
$$


is the bias-adjusted MLE $\hat{\gamma}_{M L^{*}}$, with $\hat{k}=k\left(\hat{\theta}_{M L}, X_{(1)}\right), \partial^{j} \hat{k} / \partial \theta^{j}=$ $\left(\partial^{j} k / \partial \theta^{j}\right)\left(\hat{\theta}_{M L}, X_{(1)}\right)(j=1,2), \hat{\lambda}_{j}=\lambda_{j}\left(\hat{\theta}_{M L}, X_{(1)}\right)(j=2,3)$ and $\partial \hat{\lambda}_{1} / \partial \gamma=$ $\left(\partial \lambda_{1} / \partial \gamma\right)\left(\hat{\theta}_{M L}, X_{(1)}\right)$ (see Akahira and Ohyauchi (2016a)). Then, we have

$$
k\left(T_{B, \hat{\theta}_{M L}}-T_{(1)}^{* *}\right)=\frac{B}{n}+O_{p}\left(\frac{1}{n \sqrt{n}}\right),
$$

which affects only the difference between their asymptotic means. That is, $\hat{\gamma}_{B, \hat{\theta}_{M L}}$ is second order asymptotic equivalent to the bias-adjusted MLE $\hat{\gamma}_{M L^{*}}=X_{(1)}^{* *}$, hence

$$
V_{\theta, \gamma}\left(k T_{B, \hat{\theta}_{M L}}\right)-V_{\theta, \gamma}\left(k T_{(1)}^{* *}\right)=O\left(\frac{1}{n \sqrt{n}}\right) .
$$

Further, it is easily seen from (3.7) and (4.7) the second order asymptotic loss of $\hat{\gamma}_{B, \hat{\theta}_{M L}}$ relative to $\hat{\gamma}_{B, \theta}$ is given by

$$
d_{n}\left(\hat{\gamma}_{B, \hat{\theta}_{M L}}, \hat{\gamma}_{B, \theta}\right):=n\left\{V_{\theta, \gamma}\left(k T_{B, \hat{\theta}_{M L}}\right)-V_{\gamma}\left(k T_{B, \theta}\right)\right\}=\frac{1}{\lambda_{2}}\left\{u(\gamma)-\lambda_{1}\right\}^{2}+o(1) .
$$

\section{Examples}

We can consider a truncated exponential, Pareto and a truncated normal cases as in Akahira (2016) and Akahira and Ohyauchi (2016a), but, here, we further consider a truncated Beta distribution, a truncated Erlang distribution and a truncated lognormal distribution as other examples.

Example 1 (Truncated Beta distribution). Let $c=0, d=1, a(x)=x^{-1}$ and $u(x)=\log x$ for $0<\gamma \leq x<1$ in the density (2.1). Since $b(\theta, \gamma)=$ $\theta^{-1}\left(1-\gamma^{\theta}\right)$ for $\theta>0$, it follows from (3.2), (3.4) and (4.1) that

$$
\begin{aligned}
& k(\theta, \gamma)=\theta \gamma^{\theta-1} /\left(1-\gamma^{\theta}\right), \quad \frac{\partial}{\partial \gamma} \log k(\theta, \gamma)=\frac{\theta-1}{\gamma}+\frac{\theta \gamma^{\theta-1}}{1-\gamma^{\theta}} \\
& \lambda_{1}(\theta, \gamma)=-\frac{1}{\theta}-\frac{(\log \gamma) \gamma^{\theta}}{1-\gamma^{\theta}}, \quad \lambda_{2}(\theta, \gamma)=\frac{1}{\theta^{2}}-\frac{(\log \gamma)^{2} \gamma^{\theta}}{\left(1-\gamma^{\theta}\right)^{2}} \\
& \lambda_{3}(\theta, \gamma)=-\frac{2}{\theta^{3}}-(\log \gamma)^{3} \gamma^{\theta} \frac{1+\gamma^{\theta}}{\left(1-\gamma^{\theta}\right)^{3}} .
\end{aligned}
$$

Assume that the prior density is

$$
\pi(\gamma)= \begin{cases}\alpha \gamma^{\alpha-1} & \text { for } 0<\gamma<1, \\ 0 & \text { otherwise },\end{cases}
$$

where $\alpha$ is positive and known. Let $\theta$ be known and $\hat{\gamma}_{B, \theta}$ be the Bayes estimator of $\gamma$. Since $\pi_{(1)}(\gamma)=(\alpha-1) / \gamma$ for $\gamma>0$, it is seen from (3.5)-(3.7) that the stochastic expansion, asymptotic mean and asymptotic variance of $T_{B, \theta}=$ 
$n\left(\hat{\gamma}_{B, \theta}-\gamma\right)$ are given by

$$
\begin{aligned}
& T_{B, \theta}=T_{(1)}-\frac{1-\gamma^{\theta}}{\theta \gamma^{\theta-1}}+\frac{\theta-\left(1-\gamma^{\theta}\right)}{\theta \gamma^{\theta} n} T_{(1)}+\frac{1-\gamma^{\theta}}{\theta^{2} \gamma^{2 \theta-1} n}\left\{(\alpha+1)\left(1-\gamma^{\theta}\right)-2 \theta\right\} \\
& \quad+O_{p}\left(\frac{1}{n^{2}}\right), \\
& E_{\gamma}\left(k T_{B, \theta}\right)=\frac{1}{\theta \gamma^{\theta} n}\left\{\alpha-2 \theta+1-(\alpha+1) \gamma^{\theta}\right\}+O\left(\frac{1}{n^{2}}\right), \\
& V_{\gamma}\left(k T_{B, \theta}\right)=1-\frac{2}{\theta \gamma^{\theta} n}\left\{\theta-\left(1-\gamma^{\theta}\right)\right\}+O\left(\frac{1}{n^{2}}\right),
\end{aligned}
$$

where $k=k(\theta, \gamma)$. Next, let $\theta$ be unknown and $\hat{\gamma}_{B, \hat{\theta}_{M L}}$ be the Bayes estimator plugging $\hat{\theta}_{M L}$ in $\theta$ of $\hat{\gamma}_{B, \theta}$. Since

$$
\begin{aligned}
& \frac{\partial \lambda_{1}}{\partial \gamma}=-\frac{\gamma^{\theta-1}}{\left(1-\gamma^{\theta}\right)^{2}}\left(1-\gamma^{\theta}-\theta \log \gamma\right), \\
& \frac{\partial k}{\partial \theta}=\frac{\gamma^{\theta-1}}{1-\gamma^{\theta}}\left(1+\frac{\theta \log \gamma}{1-\gamma^{\theta}}\right), \quad \frac{\partial^{2} k}{\partial \theta^{2}}=\frac{(\log \gamma) \theta \gamma^{\theta-1}}{\left(1-\gamma^{\theta}\right)^{2}}\left\{\frac{2}{\theta}+\frac{(\log \gamma)\left(1+\gamma^{\theta}\right)}{1-\gamma^{\theta}}\right\},
\end{aligned}
$$

in a similar way to the above, from (4.5)-(4.7) and (5.1)-(5.3) we obtain the stochastic expansion, asymptotic mean, and asymptotic variance of $T_{B, \hat{\theta}_{M L}}$. In particular

$$
V_{\theta, \gamma}\left(k T_{B, \hat{\theta}_{M L}}\right)=1-\frac{2}{\theta \gamma^{\theta} n}\left\{\theta-\left(1-\gamma^{\theta}\right)\right\}+\frac{1}{\lambda_{2} n}\left(\frac{1}{\theta}+\frac{\log \gamma}{1-\gamma^{\theta}}\right)^{2}+O\left(\frac{1}{n \sqrt{n}}\right),
$$

where $\lambda_{2}$ is given by (5.2).

Example 2 (Truncated Erlang distribution). Let $c=0, d=\infty, a(x)=x^{j-1}$ and $u(x)=-x$ for $0<\gamma \leq x<\infty$ in the density (2.1), where $j=1,2, \ldots$ Note that the density is truncated exponential for $j=1$. For each $j=1,2, \ldots$,

$$
b_{j}(\theta, \gamma):=\int_{\gamma}^{\infty} x^{j-1} e^{-\theta x} d x
$$

for $\theta \in \Theta=(0, \infty)$. Since $\partial b_{j} / \partial \theta=-b_{j+1}(j=1,2, \ldots)$, it follows from (3.2), (3.4) and (4.1) that, for each $j=1,2, \ldots$

$$
\begin{aligned}
& k_{j}(\theta, \gamma):=\frac{a(\gamma) e^{\theta u(\gamma)}}{b_{j}(\theta, \gamma)}=\frac{\gamma^{j-1} e^{-\theta \gamma}}{b_{j}(\theta, \gamma)}, \\
& \frac{\partial}{\partial \gamma} \log k_{j}(\theta, \gamma)=\frac{j-1}{\gamma}-\theta+k_{j}(\theta, \gamma), \\
& \lambda_{j 1}:=\frac{\partial}{\partial \theta} \log b_{j}(\theta, \gamma)=-\frac{b_{j+1}}{b_{j}} \\
& \lambda_{j 2}:=\frac{\partial^{2}}{\partial \theta^{2}} \log b_{j}(\theta, \gamma)=\frac{b_{j+2}}{b_{j}}-\left(\frac{b_{j+1}}{b_{j}}\right)^{2}, \\
& \lambda_{j 3}:=\frac{\partial^{3}}{\partial \theta^{3}} \log b_{j}(\theta, \gamma)=-\frac{b_{j+3}}{b_{j}}+\frac{3 b_{j+1} b_{j+2}}{b_{j}^{2}}-2\left(\frac{b_{j+1}}{b_{j}}\right)^{3},
\end{aligned}
$$


where $b_{j}=b_{j}(\theta, \gamma)(j=1,2, \ldots)$. Assume that the prior density is

$$
\pi(\gamma)= \begin{cases}\frac{1}{\Gamma(\alpha)} \gamma^{\alpha-1} e^{-\gamma} & \text { for } \gamma>0 \\ 0 & \text { otherwise }\end{cases}
$$

where $\alpha$ is positive and known. Let $\theta$ be known and, for each $j=1,2, \ldots$, let $\hat{\gamma}_{B, \theta}^{(j)}$ be the Bayes estimator of $\gamma$. Since

$$
\pi_{(1)}(\gamma)=\frac{\alpha-1}{\gamma}-1 \quad \text { for } \quad \gamma>0
$$

it is seen from (3.5)-(3.7) that, for each $j=1,2, \ldots$, the stochastic expansion, asymptotic mean and asymptotic variance of $T_{B, \theta}^{(j)}=n\left(\hat{\gamma}_{B, \theta}^{(j)}-\gamma\right)$ are given by

$$
\begin{aligned}
T_{B, \theta}^{(j)}= & T_{(1)}-\frac{b_{j}}{\gamma^{j-1} e^{-\theta \gamma}}+\frac{b_{j} T_{(1)}}{\gamma^{j-1} e^{-\theta \gamma} n}\left(\frac{j-1}{\gamma}-\theta+\frac{\gamma^{j-1} e^{-\theta \gamma}}{b_{j}}\right) \\
- & \frac{b_{j}^{2}}{\gamma^{2(j-1)} e^{-2 \theta \gamma} n}\left\{2\left(\frac{j-1}{\gamma}-\theta+\frac{\gamma^{j-1} e^{-\theta \gamma}}{b_{j}}\right)-\frac{\alpha-1}{\gamma}+1\right\} \\
+ & O_{p}\left(\frac{1}{n^{2}}\right), \\
E_{\gamma}\left(k_{j} T_{B, \theta}^{(j)}\right)= & -\frac{b_{j}}{\gamma^{j-1} e^{-\theta \gamma} n}\left\{2\left(\frac{j-1}{\gamma}-\theta+\frac{\gamma^{j-1} e^{-\theta \gamma}}{b_{j}}\right)-\frac{\alpha-1}{\gamma}+1\right\} \\
& +O\left(\frac{1}{n^{2}}\right), \\
V_{\gamma}\left(k_{j} T_{B, \theta}^{(j)}\right)= & 1-\frac{2 b_{j}}{\gamma^{j-1} e^{-\theta \gamma} n}\left(\frac{j-1}{\gamma}-\theta+\frac{\gamma^{j-1} e^{-\theta \gamma}}{b_{j}}\right)+O\left(\frac{1}{n^{2}}\right),
\end{aligned}
$$

where $k_{j}=k_{j}(\theta, \gamma)$ and $b_{j}=b_{j}(\theta, \gamma)$. Next, let $\theta$ be unknown and, for each $j=1,2, \ldots$, let $\hat{\gamma}_{B, \hat{\theta}_{M L}}^{(j)}$ be the Bayes estimator plugging $\hat{\theta}_{M L}$ in $\theta$ of $\hat{\gamma}_{B, \theta}^{(j)}$. Since, for each $j=1,2, \ldots$,

$$
\begin{aligned}
& \frac{\partial \lambda_{1 j}}{\partial \gamma}=\frac{\gamma^{j-1} e^{-\theta \gamma}}{b_{j}}\left(\frac{b_{j+1}}{b_{j}}+\gamma\right), \quad \frac{\partial k_{j}}{\partial \theta}=\frac{\gamma^{j-1} e^{-\theta \gamma}}{b_{j}}\left(\frac{b_{j+1}}{b_{j}}-\gamma\right), \\
& \frac{\partial^{2} k_{j}}{\partial \theta^{2}}=\frac{\gamma^{j-1} e^{-\theta \gamma}}{b_{j}}\left\{\left(\frac{b_{j+1}}{b_{j}}\right)^{2}+\left(\frac{b_{j+1}}{b_{j}}-\gamma\right)^{2}-\frac{b_{j+2}}{b_{j}}\right\},
\end{aligned}
$$

in a similar way to the above, from (4.5)-(4.7) and (5.4)-(5.6) we obtain the stochastic expansion, asymptotic mean and asymptotic variance of $T_{B, \hat{\theta}_{M L}}^{(j)}$ for each $j=1,2, \ldots$. In particular,

$$
\begin{aligned}
V_{\theta, \gamma}\left(k_{j} T_{B, \hat{\theta}_{M L}}^{(j)}\right)= & 1-\frac{2 b_{j}}{\gamma^{j-1} e^{-\theta \gamma}}\left(\frac{j-1}{\gamma}-\theta+\frac{\gamma^{j-1} e^{-\theta \gamma}}{b_{j}}\right) \\
& +\frac{b_{j}^{2}}{\left(b_{j} b_{j+2}-b_{j+1}^{2}\right) n}\left(\frac{b_{j+1}}{b_{j}}-\gamma\right)^{2}+O\left(\frac{1}{n \sqrt{n}}\right) .
\end{aligned}
$$


Example 3 (Truncated lognormal distribution). Let $c=0, d=\infty, a(x)=$ $x^{-1} \exp \left\{-(1 / 2)(\log x)^{2}\right\}$ and $u(x)=\log x$ for $0<\gamma \leq x<\infty$ in the density $(2.1)$. Then, $b(\theta, \gamma)=\Phi(\theta-\log \gamma) / \phi(\theta)$ for $\theta \in \Theta=(-\infty, \infty)$, where $\Phi(x)=\int_{-\infty}^{x} \phi(t) d t$ with $\phi(t)=(1 / \sqrt{2 \pi}) e^{-t^{2} / 2}$ for $-\infty<t<\infty$. Letting $t=\log x$ and $\gamma_{0}=\log \gamma$, we see that $(2.1)$ becomes

$$
f\left(t ; \theta, \gamma_{0}\right)= \begin{cases}\frac{1}{\sqrt{2 \pi} \Phi\left(\theta-\gamma_{0}\right)} e^{-(t-\theta)^{2} / 2} & \text { for }-\infty<\gamma_{0} \leq t<\infty \\ 0 & \text { otherwise }\end{cases}
$$

Hence, the truncated lognormal case is reduced to the truncated normal one in Example 1 of Akahira (2016) and Example 6.3 of Akahira and Ohyauchi (2016a). In this case, the standard normal distribution may be taken as a prior one.

For a truncated Beta distribution and a truncated Erlang distribution, related results to the above can be found in Vancak et al. (2015).

\section{Concluding remarks}

For a one-sided truncated exponential family $\mathcal{P}$ of distributions with a truncation parameter $\gamma$ and a natural parameter $\theta$, we considered the estimation problem on $\gamma$ in the presence of $\theta$ as a nuisance parameter from the Bayesian viewpoint. Under a smooth prior density $\pi(\gamma)$ of $\gamma$ and a quadratic loss, the stochastic expansions of the Bayes estimator $\hat{\gamma}_{B, \theta}$ (i.e., (2.3)) of $\gamma$ when $\theta$ was known and the Bayes estimator $\hat{\gamma}_{B, \hat{\theta}_{M L}}$ (i.e., (4.4)) plugging the MLE $\hat{\theta}_{M L}$ in $\theta$ of $\hat{\gamma}_{B, \theta}$ when $\theta$ was unknown, were derived, which led to the fact that the asymptotic means of $T_{B, \theta}=n\left(\hat{\gamma}_{B, \theta}-\gamma\right)$ and $T_{B, \hat{\theta}_{M L}}=n\left(\hat{\gamma}_{B, \hat{\theta}_{M L}}-\gamma\right)$ depended on the prior $\pi$, but their asymptotic variances were independent of it. In the previous discussion, we adopted a partial Bayesian approach; to be precise, we chose the combined Bayesian-frequentist approach. Indeed, since a density (2.1) in the truncated exponential family $\mathcal{P}$ has a truncation point $\gamma$, it is considered to be helpful to obtain some information through a prior on $\gamma$. On the other hand, a natural parameter $\theta$ in $\mathcal{P}$ is in the same situation as in a regular exponential family, hence the maximum likelihood method based on the likelihood equation is useful for estimating $\theta$. Hence it seems to be natural to plug the MLE in $\theta$ of the Bayes estimator of $\gamma$ for known $\theta$. But, taking a pure Bayesian approach, one may obtain the Bayes estimator with respect to a prior on $(\theta, \gamma)$. It seems to be interesting to compare it with our estimator.

Recently, for a two-sided truncated exponential family of distributions with a natural parameter $\theta$ and two truncation parameters $\gamma$ and $\nu$, the ML estimation of $\theta$ when $\gamma$ and $\nu$ are nuisance parameters has been discussed by Akahira et al. (2016) and the ML estimation of $\gamma$ or $\nu$ when $\theta$ is a nuisance parameter has been also discussed by Akahira and Ohyauchi (2016b). Such a family includes the truncated Pareto distribution which is widely used in finance, hydrology, and atmospheric science (see Aban et al. (2006)). In this case, we may also obtain the Bayes estimator with respect to a prior on $(\theta, \gamma, \nu)$ and compare it with the partial Bayes estimator. 


\section{Appendix}

For the proofs of Theorems 1 and 2, we need the following theorems.

Theorem A.1 (Akahira and Ohyauchi (2016a)). Let $X_{(1)}^{*}$ be the biasadjusted $M L E \hat{\gamma}_{M L^{*}}^{\theta}$ of $\gamma$ when $\theta$ is known (see Remark 1). Then the stochastic expansion of $T_{(1)}^{*}=n\left(X_{(1)}^{*}-\gamma\right)$ is given by the part except the term (3.8) in the right-hand side of (3.5), and the second order asymptotic mean and variance are given by

$$
\begin{aligned}
& E_{\theta, \gamma}\left[T_{(1)}^{*}\right]=O\left(\frac{1}{n^{2}}\right), \\
& V_{\gamma}\left(k T_{(1)}^{*}\right)=1-\frac{2}{k n}\left(\frac{\partial}{\partial \gamma} \log k\right)+O\left(\frac{1}{n^{2}}\right),
\end{aligned}
$$

respectively, where $k=k(\theta, \gamma)$.

Theorem A.2 (Akahira and Ohyauchi (2016a)). Let $X_{(1)}^{* *}$ be the biasadjusted $M L E \hat{\gamma}_{M L^{*}}$ of $\gamma$ when $\theta$ is unknown (see Remark 3 ). Then the stochastic expansion of $T_{(1)}^{* *}=n\left(X_{(1)}^{* *}-\gamma\right)$ is given by the part except the term $B /(k n)$ in the right-hand side of (4.5), and the second order asymptotic mean and variance are given by

$$
\begin{aligned}
& E_{\theta, \gamma}\left[T_{(1)}^{* *}\right]=O\left(\frac{1}{n \sqrt{n}}\right) \\
& V_{\theta, \gamma}\left(k T_{(1)}^{* *}\right)=1-\frac{2}{k n}\left(\frac{\partial}{\partial \gamma} \log k\right)+\frac{1}{\lambda_{2} n}\left(u(\gamma)-\lambda_{1}\right)^{2}+O\left(\frac{1}{n \sqrt{n}}\right)
\end{aligned}
$$

respectively, where $k=k(\theta, \gamma)$.

The Proof of Theorem 1. Since, by the Taylor expansion,

$$
\begin{aligned}
b\left(\theta, \gamma+\frac{v}{n}\right)=b(\theta, \gamma)\{ & 1+\left(\frac{\partial}{\partial \gamma} \log b(\theta, \gamma)\right) \frac{v}{n} \\
& \left.+\frac{1}{2 b(\theta, \gamma)}\left(\frac{\partial^{2} b(\theta, \gamma)}{\partial \gamma^{2}}\right) \frac{v^{2}}{n^{2}}+O\left(\frac{1}{n^{3}}\right)\right\}
\end{aligned}
$$

as $n \rightarrow \infty$, it follows that

$$
\begin{aligned}
b^{n}\left(\theta, \gamma+\frac{v}{n}\right)=b^{n}(\theta, \gamma) \exp \{ & b_{(1)} v-\frac{1}{2 n} b_{(1)}^{2} v^{2} \\
+ & \left.\frac{1}{2 b n}\left(\frac{\partial^{2} b}{\partial \gamma^{2}}\right) v^{2}+O\left(\frac{1}{n^{2}}\right)\right\}
\end{aligned}
$$

where $b=b(\theta, \gamma)$ and $b_{(j)}=b_{(j)}(\theta, \gamma)$. Here

$$
\frac{1}{b(\theta, \gamma)} \frac{\partial^{2}}{\partial \gamma^{2}} b(\theta, \gamma)=b_{(2)}+b_{(1)}^{2} .
$$


Substituting (A.6) into (A.5) we have

$$
b^{n}\left(\theta, \gamma+\frac{v}{n}\right)=b^{n}(\theta, \gamma) e^{b_{(1)} v}\left\{1+\frac{b_{(2)}}{2 n} v^{2}+O\left(\frac{1}{n^{2}}\right)\right\} .
$$

From (3.3), we have

$$
\pi\left(\gamma+\frac{v}{n}\right)=\pi(\gamma)\left\{1+\pi_{(1)} \frac{v}{n}+O\left(\frac{1}{n^{2}}\right)\right\},
$$

where $\pi_{(1)}=\pi_{(1)}(\gamma)$. From (A.7) and (A.8) we obtain

$$
\frac{\pi\left(\gamma+\frac{v}{n}\right)}{b^{n}\left(\theta, \gamma+\frac{v}{n}\right)}=\frac{\pi(\gamma) e^{-b_{(1)} v}}{b^{n}(\theta, \gamma)}\left\{1-\frac{b_{(2)}}{2 n} v^{2}+\frac{\pi_{(1)}}{n} v+O\left(\frac{1}{n^{2}}\right)\right\}
$$

Putting $I_{j}:=\int_{\tau_{n}}^{T_{(1)}} v^{j} e^{k v} d v(j=0,1,2,3)$, we have from (3.4) and (A.9)

$$
\begin{aligned}
& \int_{\tau_{n}}^{T_{(1)}} \frac{\pi\left(\gamma+\frac{v}{n}\right)}{b^{n}\left(\theta, \gamma+\frac{v}{n}\right)} d v=\frac{\pi(\gamma)}{b^{n}(\theta, \gamma)}\left\{I_{0}-\frac{b_{(2)}}{2 n} I_{2}+\frac{\pi_{(1)}}{n} I_{1}+O_{p}\left(\frac{1}{n^{2}}\right)\right\} \\
& \int_{\tau_{n}}^{T_{(1)}} \frac{v \pi\left(\gamma+\frac{v}{n}\right)}{b^{n}\left(\theta, \gamma+\frac{v}{n}\right)} d v=\frac{\pi(\gamma)}{b^{n}(\theta, \gamma)}\left\{I_{1}-\frac{b_{(2)}}{2 n} I_{3}+\frac{\pi_{(1)}}{n} I_{2}+O_{p}\left(\frac{1}{n^{2}}\right)\right\}
\end{aligned}
$$

Here, the remainder terms in (A.10) and (A.11) are guaranteed to be order $O_{p}\left(n^{-2}\right)$, since the distribution with a density (2.1) belongs to a truncated exponential family $\mathcal{P}$ with a normalizing factor $b(\theta, \gamma)$ based on $a(\cdot)$ and $u(\cdot)$ which are functions of class $\mathbb{C}^{3}$. From (A.10) and (A.11) we obtain

$$
\begin{aligned}
\int_{\tau_{n}}^{T_{(1)}} & \frac{v \pi\left(\gamma+\frac{v}{n}\right)}{b^{n}\left(\theta, \gamma+\frac{v}{n}\right)} d v / \int_{\tau_{n}}^{T_{(1)}} \frac{\pi\left(\gamma+\frac{v}{n}\right)}{b^{n}\left(\theta, \gamma+\frac{v}{n}\right)} d v \\
= & \left\{I_{1}-\frac{b_{(2)}}{2 n} I_{3}+\frac{\pi_{(1)}}{n} I_{2}+O_{p}\left(\frac{1}{n^{2}}\right)\right\} \\
& \times\left\{I_{0}-\frac{b_{(2)}}{2 n} I_{2}+\frac{\pi_{(1)}}{n} I_{1}+O_{p}\left(\frac{1}{n^{2}}\right)\right\}^{-1} \\
= & \frac{I_{1}}{I_{0}}+\frac{b_{(2)}}{2 n}\left(\frac{I_{1} I_{2}}{I_{0}^{2}}-\frac{I_{3}}{I_{0}}\right)-\frac{\pi_{(1)}}{n}\left\{\left(\frac{I_{1}}{I_{0}}\right)^{2}-\frac{I_{2}}{I_{0}}\right\}+O_{p}\left(\frac{1}{n^{2}}\right) .
\end{aligned}
$$

Substituting (A.12) into (3.1), we have

$$
\begin{aligned}
T_{B, \theta} & :=n\left(\hat{\gamma}_{B, \theta}-\gamma\right) \\
& =\frac{I_{1}}{I_{0}}+\frac{b_{(2)}}{2 n}\left(\frac{I_{1} I_{2}}{I_{0}^{2}}-\frac{I_{3}}{I_{0}}\right)-\frac{\pi_{(1)}}{n}\left\{\left(\frac{I_{1}}{I_{0}}\right)^{2}-\frac{I_{2}}{I_{0}}\right\}+O_{p}\left(\frac{1}{n^{2}}\right) .
\end{aligned}
$$


Since

$$
\begin{aligned}
I_{0} & =\int_{\tau_{n}}^{T_{(1)}} e^{k v} d v=\frac{1}{k} e^{k T_{(1)}}+O_{p}\left(e^{k \tau_{n}}\right) \\
I_{1} & =\int_{\tau_{n}}^{T_{(1)}} v e^{k v} d v=\frac{1}{k} e^{k T_{(1)}}\left(T_{(1)}-\frac{1}{k}\right)+O_{p}\left(n e^{k \tau_{n}}\right) \\
I_{2} & =\int_{\tau_{n}}^{T_{(1)}} v^{2} e^{k v} d v=\frac{1}{k} e^{k T_{(1)}}\left\{\left(T_{(1)}-\frac{1}{k}\right)^{2}+\frac{1}{k^{2}}\right\}+O_{p}\left(n^{2} e^{k \tau_{n}}\right), \\
I_{3} & =\int_{\tau_{n}}^{T_{(1)}} v^{3} e^{k v} d v \\
& =\frac{1}{k} e^{k T_{(1)}}\left\{\left(T_{(1)}-\frac{1}{k}\right)^{3}+\frac{3}{k^{2}}\left(T_{(1)}-\frac{1}{k}\right)-\frac{2}{k^{3}}\right\}+O_{p}\left(n^{3} e^{k \tau_{n}}\right)
\end{aligned}
$$

as $n \rightarrow \infty$, it follows that

$$
\frac{I_{1}}{I_{0}}=T_{(1)}-\frac{1}{k}+O_{p}\left(e^{k\left(\tau_{n}-T_{(1)}\right)}\right),
$$

$$
\begin{aligned}
& \frac{I_{2}}{I_{0}}=\left(T_{(1)}-\frac{1}{k}\right)^{2}+\frac{1}{k^{2}}+O_{p}\left(n^{2} e^{k\left(\tau_{n}-T_{(1)}\right)}\right), \\
& \frac{I_{3}}{I_{0}}=\left(T_{(1)}-\frac{1}{k}\right)^{3}+\frac{3}{k^{2}}\left(T_{(1)}-\frac{1}{k}\right)-\frac{2}{k^{3}}+O_{p}\left(n^{3} e^{k\left(\tau_{n}-T_{(1)}\right)}\right),
\end{aligned}
$$

hence, by (A.13)

$$
T_{B, \theta}=T_{(1)}-\frac{1}{k}-\frac{b_{(2)}}{k^{2} n} T_{(1)}+\frac{1}{k^{2} n}\left(\frac{2 b_{(2)}}{k}+\pi_{(1)}\right)+O_{p}\left(\frac{1}{n^{2}}\right) .
$$

Since by (3.2) and (3.4)

$$
\frac{b_{(2)}}{k}=\frac{1}{k}\left(\frac{\partial^{2}}{\partial \gamma^{2}} \log b\right)=-\frac{1}{k}\left(\frac{\partial k}{\partial \gamma}\right)=-\frac{\partial}{\partial \gamma} \log k
$$

it follows from (A.17) and (A.18) that

$$
\begin{aligned}
T_{B, \theta}= & T_{(1)}-\frac{1}{k}+\frac{1}{k n}\left(\frac{\partial}{\partial \gamma} \log k\right) T_{(1)} \\
& -\frac{1}{k^{2} n}\left\{2\left(\frac{\partial}{\partial \gamma} \log k\right)-\pi_{(1)}\right\}+O_{p}\left(\frac{1}{n^{2}}\right)
\end{aligned}
$$

which implies that (3.5) holds. From (3.5) and Theorem A.1 we have

$$
T_{B, \theta}=T_{(1)}^{*}-\frac{1}{k^{2} n}\left\{2\left(\frac{\partial}{\partial \gamma} \log k\right)-\pi_{(1)}\right\}+O_{p}\left(\frac{1}{n^{2}}\right),
$$

hence, by (A.1) and (A.2) we obtain (3.6) and (3.7). Thus we complete the proof. 
The Proof of Theorem 2. The Bayes estimator (4.4) plugging $\hat{\theta}_{M L}$ in $\theta$ of $\hat{\gamma}_{B, \theta}$ when $\theta$ is unknown, is expressed by

$$
\begin{array}{r}
\hat{\gamma}_{B, \hat{\theta}_{M L}}(\boldsymbol{X})=\gamma+\frac{1}{n}\left(\int_{\tau_{n}}^{T_{(1)}} \frac{u \pi\left(\gamma+\frac{u}{n}\right)}{b^{n}\left(\hat{\theta}_{M L}, \gamma+\frac{u}{n}\right)} d u /\right. \\
\left.\int_{\tau_{n}}^{T_{(1)}} \frac{\pi\left(\gamma+\frac{u}{n}\right)}{b^{n}\left(\hat{\theta}_{M L}, \gamma+\frac{u}{n}\right)} d u\right) .
\end{array}
$$

Since $\hat{\theta}_{M L}=\theta+\left(\hat{U} / \sqrt{\lambda_{2} n}\right)$ and

$$
\begin{aligned}
& \frac{1}{\lambda(\theta, \gamma)} \frac{\partial^{2}}{\partial \theta^{2}} b(\theta, \gamma)=\lambda_{2}+\lambda_{1}^{2}, \\
& \frac{1}{\lambda(\theta, \gamma)} \frac{\partial^{3}}{\partial \theta^{3}} b(\theta, \gamma)=\lambda_{3}+3 \lambda_{1} \lambda_{2}+\lambda_{1}^{3},
\end{aligned}
$$

we have by the Taylor expansion

$$
\begin{aligned}
b\left(\theta+\frac{\hat{U}}{\sqrt{\lambda_{2} n}}, \gamma+\frac{u}{n}\right) & \\
=b(\theta, \gamma)\{ & 1+\frac{\lambda_{1}}{\sqrt{\lambda_{2} n}} \hat{U}+\frac{b_{(1)}}{n} u+\frac{\lambda_{2}+\lambda_{1}^{2}}{2 \lambda_{2} n} \hat{U}^{2}+\frac{1}{b}\left(\frac{\partial^{2} b}{\partial \theta \partial \gamma}\right) \frac{\hat{U} u}{\sqrt{\lambda_{2}} n \sqrt{n}} \\
& +\frac{\lambda_{3}+3 \lambda_{1} \lambda_{2}+\lambda_{1}^{3}}{6 \lambda_{2}^{3 / 2} n \sqrt{n}} \hat{U}^{3}+\frac{1}{2 b \lambda_{2} n^{2}}\left(\frac{\partial^{3} b}{\partial \theta^{2} \partial \gamma}\right) \hat{U}^{2} u \\
& \left.+\frac{1}{2 b n^{2}}\left(\frac{\partial^{2} b}{\partial \gamma^{2}}\right) u^{2}+\frac{1}{24 b \lambda_{2}^{2} n^{2}}\left(\frac{\partial^{4} b}{\partial \theta^{4}}\right) \hat{U}^{4}+O\left(\frac{1}{n^{2} \sqrt{n}}\right)\right\},
\end{aligned}
$$

hence

$$
\begin{aligned}
&\left(\text { A.20 } b^{n}\left(\theta+\frac{\hat{U}}{\sqrt{\lambda_{2} n}}, \gamma+\frac{u}{n}\right)\right. \\
&=b^{n}(\theta, \gamma)\left[\operatorname { e x p } \left\{\frac{\lambda_{1} \sqrt{n}}{\sqrt{\lambda_{2}}} \hat{U}+\frac{1}{2} \hat{U}^{2}+\frac{\lambda_{3}}{6 \lambda_{2}^{3 / 2} \sqrt{n}} \hat{U}^{3}\right.\right. \\
&+\frac{1}{24 b \lambda_{2}^{2} n}\left(\frac{\partial^{4} b}{\partial \theta^{4}}\right) \hat{U}^{4} \\
&\left.\left.-\frac{\lambda_{1}^{4}+6 \lambda_{1}^{2} \lambda_{2}+4 \lambda_{1} \lambda_{3}+3 \lambda_{2}^{2}}{24 \lambda_{2}^{2} n} \hat{U}^{4}\right\}\right] \\
& \cdot e^{b_{(1)} u} \exp \left\{\frac{\hat{U}}{b \sqrt{\lambda_{2} n}}\left(\frac{\partial^{2} b}{\partial \theta \partial \gamma}\right) u+\frac{b_{(2)}}{2 n} u^{2}+\frac{\hat{U}^{2}}{2 b \lambda_{2} n}\left(\frac{\partial^{3} b}{\partial \theta^{2} \partial \gamma}\right) u\right. \\
&-\frac{\lambda_{1} b_{(1)}}{\sqrt{\lambda_{2} n}} u-\frac{b_{(1)}\left(\lambda_{2}+\lambda_{1}^{2}\right) \hat{U}^{2}}{2 \lambda_{2} n} u \\
&\left.-\frac{\lambda_{1}}{b \lambda_{2} n}\left(\frac{\partial^{2} b}{\partial \theta \partial \gamma}\right) \hat{U}^{2} u+\frac{b_{(1)} \lambda_{1}^{2}}{\lambda_{2} n} \hat{U}^{2} u+O_{p}\left(\frac{1}{n \sqrt{n}}\right)\right\}
\end{aligned}
$$




$$
\begin{gathered}
=: b^{n}(\theta, \gamma)[\exp \{Q\}] \\
\cdot e^{b_{(1)} u} \exp \left\{\frac{\hat{U}}{b \sqrt{\lambda_{2} n}}\left(\frac{\partial^{2} b}{\partial \theta \partial \gamma}\right) u+\frac{b_{(2)}}{2 n} u^{2}+\frac{\hat{U}^{2}}{2 b \lambda_{2} n}\left(\frac{\partial^{3} b}{\partial \theta^{2} \partial \gamma}\right) u\right. \\
\quad-\frac{\lambda_{1} b_{(1)} \hat{U}}{\sqrt{\lambda_{2} n}} u-\frac{b_{(1)}\left(\lambda_{2}-\lambda_{1}^{2}\right) \hat{U}^{2}}{2 \lambda_{2} n} u \\
\left.\quad-\frac{\lambda_{1}}{b \lambda_{2} n}\left(\frac{\partial^{2} b}{\partial \theta \partial \gamma}\right) \hat{U}^{2} u+O_{p}\left(\frac{1}{n \sqrt{n}}\right)\right\},
\end{gathered}
$$

where $Q$ is independent of $u$. From (A.8) and (A.20) we have

$$
\begin{aligned}
& \frac{\pi\left(\gamma+\frac{u}{n}\right)}{b^{n}\left(\theta+\frac{\hat{U}}{\sqrt{\lambda_{2} n}}, \gamma+\frac{u}{n}\right)} \\
& =\pi(\gamma)\left\{1+\frac{\pi_{(1)}}{n} u+O\left(\frac{1}{n^{2}}\right)\right\} \cdot b^{-n}(\theta, \gamma)[\exp \{-Q\}] e^{-b_{(1)} u} \\
& \cdot \exp \left\{-\frac{\hat{U}}{b \sqrt{\lambda_{2} n}}\left(\frac{\partial^{2} b}{\partial \theta \partial \gamma}\right) u-\frac{b_{(2)}}{2 n} u^{2}-\frac{\hat{U}^{2}}{2 b \lambda_{2} n}\left(\frac{\partial^{3} b}{\partial \theta^{2} \partial \gamma}\right) u\right. \\
& +\frac{\lambda_{1} b_{(1)} \hat{U}}{\sqrt{\lambda_{2} n}} u+\frac{b_{(1)}\left(\lambda_{2}-\lambda_{1}^{2}\right) \hat{U}^{2}}{2 \lambda_{2} n} u \\
& \left.+\frac{\lambda_{1}}{b \lambda_{2} n}\left(\frac{\partial^{2} b}{\partial \theta \partial \gamma}\right) \hat{U}^{2} u+O_{p}\left(\frac{1}{n \sqrt{n}}\right)\right\} \\
& =b^{-n}(\theta, \gamma)[\exp \{-Q\}] \pi(\gamma) e^{-b_{(1)} u} \\
& \cdot\left[1-\frac{\hat{U}}{b \sqrt{\lambda_{2} n}}\left(\frac{\partial^{2} b}{\partial \theta \partial \gamma}\right) u+\frac{\lambda_{1} b_{(1)}}{\sqrt{\lambda_{2} n}} \hat{U} u-\frac{b_{(2)}}{2 n} u^{2}\right. \\
& +\frac{b_{(1)}\left(\lambda_{2}-\lambda_{1}^{2}\right)}{2 \lambda_{2} n} \hat{U}^{2} u-\frac{\hat{U}^{2}}{2 b \lambda_{2} n}\left(\frac{\partial^{3} b}{\partial \theta^{2} \partial \gamma}\right) u+\frac{\lambda_{1}}{b \lambda_{2} n}\left(\frac{\partial^{2} b}{\partial \theta \partial \gamma}\right) \hat{U}^{2} u \\
& +\frac{\pi_{(1)}}{n} u+\frac{1}{2}\left\{\frac{1}{b^{2}}\left(\frac{\partial^{2} b}{\partial \theta \partial \gamma}\right)^{2} \frac{1}{\lambda_{2} n} \hat{U}^{2} u^{2}-\frac{2 \lambda_{1} b_{(1)} \hat{U}^{2}}{b \lambda_{2} n}\left(\frac{\partial^{2} b}{\partial \theta \partial \gamma}\right) u^{2}\right. \\
& \left.\left.+\frac{\lambda_{1}^{2} b_{(1)}^{2}}{\lambda_{2} n} \hat{U}^{2} u^{2}\right\}+O_{p}\left(\frac{1}{n \sqrt{n}}\right)\right] \text {, }
\end{aligned}
$$

hence

$$
\begin{aligned}
\int_{\tau_{n}}^{T_{(1)}} & \frac{\pi\left(\gamma+\frac{u}{n}\right)}{b^{n}\left(\theta+\frac{\hat{U}}{\sqrt{\lambda_{2} n}}, \gamma+\frac{u}{n}\right)} d u \\
= & b^{-n}(\theta, \gamma)[\exp \{-Q\}] \pi(\gamma) \\
& \cdot\left[I_{0}+\frac{\hat{U}}{\sqrt{\lambda_{2} n}}\left\{\lambda_{1} b_{(1)}-\frac{1}{b}\left(\frac{\partial^{2} b}{\partial \theta \partial \gamma}\right)\right\} I_{1}-\frac{b_{(2)}}{2 n} I_{2}\right.
\end{aligned}
$$




$$
\begin{aligned}
& +\frac{b_{(1)}\left(\lambda_{2}-\lambda_{1}^{2}\right) \hat{U}^{2}}{2 \lambda_{2} n} I_{1} \\
& -\frac{1}{\lambda_{2} n}\left\{\frac{1}{2 b}\left(\frac{\partial^{3} b}{\partial \theta^{2} \partial \gamma}\right)-\frac{\lambda_{1}}{b}\left(\frac{\partial^{2} b}{\partial \theta \partial \gamma}\right)\right\} \hat{U}^{2} I_{1} \\
& +\frac{\hat{U}^{2} I_{2}}{\lambda_{2} n}\left\{\frac{1}{2 b^{2}}\left(\frac{\partial^{2} b}{\partial \theta \partial \gamma}\right)^{2}-\frac{\lambda_{1} b_{(1)}}{b}\left(\frac{\partial^{2} b}{\partial \theta \partial \gamma}\right)+\frac{1}{2} \lambda_{1}^{2} b_{(1)}^{2}\right\} \\
& \left.+\frac{\pi_{(1)}}{n} I_{1}+O_{p}\left(\frac{1}{n \sqrt{n}}\right)\right] \text {, } \\
& \int_{\tau_{n}}^{T_{(1)}} \frac{u \pi\left(\gamma+\frac{u}{n}\right)}{b^{n}\left(\theta+\frac{\hat{U}}{\sqrt{\lambda_{2} n}}, \gamma+\frac{u}{n}\right)} d u \\
& =b^{-n}(\theta, \gamma)[\exp \{-Q\}] \pi(\gamma) \\
& \cdot\left[I_{1}+\frac{\hat{U}}{\sqrt{\lambda_{2} n}}\left\{\lambda_{1} b_{(1)}-\frac{1}{b}\left(\frac{\partial^{2} b}{\partial \theta \partial \gamma}\right)\right\} I_{2}-\frac{b_{(2)}}{2 n} I_{3}\right. \\
& +\frac{b_{(1)}\left(\lambda_{2}-\lambda_{1}^{2}\right) \hat{U}^{2}}{2 \lambda_{2} n} I_{2} \\
& -\frac{1}{\lambda_{2} n}\left\{\frac{1}{2 b}\left(\frac{\partial^{3} b}{\partial \theta^{2} \partial \gamma}\right)-\frac{\lambda_{1}}{b}\left(\frac{\partial^{2} b}{\partial \theta \partial \gamma}\right)\right\} \hat{U}^{2} I_{2} \\
& +\frac{\hat{U}^{2} I_{3}}{\lambda_{2} n}\left\{\frac{1}{2 b^{2}}\left(\frac{\partial^{2} b}{\partial \theta \partial \gamma}\right)^{2}-\frac{\lambda_{1} b_{(1)}}{b}\left(\frac{\partial^{2} b}{\partial \theta \partial \gamma}\right)+\frac{1}{2} \lambda_{1}^{2} b_{(1)}^{2}\right\} \\
& \left.+\frac{\pi_{(1)}}{n} I_{2}+O_{p}\left(\frac{1}{n \sqrt{n}}\right)\right] \text {. }
\end{aligned}
$$

Here, the remainder terms in (A.21) and (A.22) are guaranteed to be order $O_{p}\left(n^{-3 / 2}\right)$, since the distribution with a density (2.1) belongs to a truncated exponential family $\mathcal{P}$ with a normalizing factor $b(\theta, \gamma)$ based on $a(\cdot)$ and $u(\cdot)$ which are functions of class $\mathbb{C}^{3}$. Substituting (A.21) and (A.22) into (A.19), we have

$$
\begin{aligned}
n\left(\hat{\gamma}_{B, \hat{\theta}_{M L}}-\gamma\right) & =\frac{I_{1}}{I_{0}}+\frac{\hat{U}}{\sqrt{\lambda_{2} n}}\left\{\lambda_{1} b_{(1)}-\frac{1}{b}\left(\frac{\partial^{2} b}{\partial \theta \partial \gamma}\right)\right\}\left\{\frac{I_{2}}{I_{0}}-\left(\frac{I_{1}}{I_{0}}\right)^{2}\right\} \\
& -\frac{1}{2 n} b_{(2)}\left(\frac{I_{3}}{I_{0}}-\frac{I_{1} I_{2}}{I_{0}^{2}}\right)+\frac{b_{(1)}\left(\lambda_{2}-\lambda_{1}^{2}\right)}{2 \lambda_{2} n} \hat{U}^{2}\left\{\frac{I_{2}}{I_{0}}-\left(\frac{I_{1}}{I_{0}}\right)^{2}\right\} \\
& \left.-\frac{\hat{U}^{2}}{\lambda_{2} n}\left\{\frac{1}{2 b}\left(\frac{\partial^{3} b}{\partial \theta^{2} \partial \gamma}\right)-\frac{\lambda_{1}}{b}\left(\frac{\partial^{2} b}{\partial \theta \partial \gamma}\right)\right\} \frac{I_{2}}{I_{0}}-\left(\frac{I_{1}}{I_{0}}\right)^{2}\right\} \\
& +\frac{\hat{U}^{2}}{\lambda_{2} n}\left\{\frac{1}{2 b^{2}}\left(\frac{\partial^{2} b}{\partial \theta \partial \gamma}\right)^{2}-\frac{\lambda_{1} b_{(1)}}{b}\left(\frac{\partial^{2} b}{\partial \theta \partial \gamma}\right)+\frac{1}{2} \lambda_{1}^{2} b_{(1)}^{2}\right\}
\end{aligned}
$$




$$
\begin{aligned}
& \cdot\left(\frac{I_{3}}{I_{0}}-\frac{I_{1} I_{2}}{I_{0}^{2}}\right)+\frac{\pi_{(1)}}{n}\left\{\frac{I_{2}}{I_{0}}-\left(\frac{I_{1}}{I_{0}}\right)^{2}\right\} \\
& +\frac{\hat{U}^{2}}{\lambda_{2} n}\left\{\lambda_{1} b_{(1)}-\frac{1}{b}\left(\frac{\partial^{2} b}{\partial \theta \partial \gamma}\right)\right\}^{2}\left(\frac{I_{1}}{I_{0}}\right)^{3} \\
& -\frac{\hat{U}^{2}}{\lambda_{2} n}\left\{\lambda_{1} b_{(1)}-\frac{1}{b}\left(\frac{\partial^{2} b}{\partial \theta \partial \gamma}\right)\right\}^{2} \frac{I_{1} I_{2}}{I_{0}^{2}}+O_{p}\left(\frac{1}{n \sqrt{n}}\right) .
\end{aligned}
$$

From (A.14)-(A.16) and (A.23) we obtain

$$
\begin{aligned}
& \text { (A.24) } n\left(\hat{\gamma}_{B, \hat{\theta}_{M L}}-\gamma\right) \\
& =T_{(1)}+\frac{1}{b_{(1)}}+\frac{1}{b_{(1)}^{2} \sqrt{\lambda_{2} n}}\left\{\lambda_{1} b_{(1)}-\frac{1}{b}\left(\frac{\partial^{2} b}{\partial \theta \partial \gamma}\right)\right\} \hat{U} \\
& -\frac{b_{(2)}}{b_{(1)}^{2} n}\left(T_{(1)}+\frac{2}{b_{(1)}}\right) \\
& +\frac{\left(\lambda_{2}-\lambda_{1}^{2}\right)}{2 b_{(1)} \lambda_{2} n} \hat{U}^{2}-\frac{1}{b_{(1)}^{2} \lambda_{2} n}\left\{\frac{1}{2 b}\left(\frac{\partial^{3} b}{\partial \theta^{2} \partial \gamma}\right)-\frac{\lambda_{1}}{b}\left(\frac{\partial^{2} b}{\partial \theta \partial \gamma}\right)\right\} \hat{U}^{2} \\
& +\frac{1}{b_{(1)}^{3} \lambda_{2} n}\left\{\lambda_{1} b_{(1)}-\frac{1}{b}\left(\frac{\partial^{2} b}{\partial \theta \partial \gamma}\right)\right\}^{2} \hat{U}^{2}+\frac{\pi_{(1)}}{b_{(1)}^{2} n}+O_{p}\left(\frac{1}{n \sqrt{n}}\right) \text {. }
\end{aligned}
$$

Here, we have from (2.2), (3.2) and (4.1)

$$
\frac{\partial^{2} b}{\partial \theta \partial \gamma}=u(\gamma) b b_{(1)}, \quad \frac{\partial^{2} b}{\partial \theta^{2}}=b\left(\lambda_{2}+\lambda_{1}^{2}\right)
$$

which imply

$$
\frac{1}{b} \frac{\partial^{3} b}{\partial \theta^{2} \partial \gamma}=\frac{\partial \lambda_{2}}{\partial \gamma}+2 \lambda_{1} u(\gamma) b_{(1)}+b_{(1)}\left(\lambda_{2}-\lambda_{1}^{2}\right)
$$

Substituting (A.25) and (A.26) into (A.24) we have from (3.4)

$$
\begin{aligned}
T_{B, \hat{\theta}_{M L}}= & n\left(\hat{\gamma}_{B, \hat{\theta}_{M L}}-\gamma\right) \\
= & T_{(1)}-\frac{1}{k}+\frac{\xi}{k \sqrt{\lambda_{2} n}} \hat{U}-\frac{b_{(2)}}{k^{2} n}\left(T_{(1)}-\frac{2}{k}\right)-\frac{\xi^{2}}{k \lambda_{2} n} \hat{U}^{2} \\
& -\frac{1}{2 k^{2} \lambda_{2} n}\left(\frac{\partial \lambda_{2}}{\partial \gamma}\right) \hat{U}^{2}+\frac{\pi_{(1)}}{k^{2} n}+O_{p}\left(\frac{1}{n \sqrt{n}}\right) .
\end{aligned}
$$

Since

$$
\xi=u(\gamma)-\lambda_{1}=\frac{\partial}{\partial \theta} \log k=\frac{1}{k}\left(\frac{\partial k}{\partial \theta}\right),
$$

it follows from (A.27) and (3.4) that

$$
\text { (A.28) } T_{B, \hat{\theta}_{M L}}=T_{(1)}-\frac{1}{k}+\frac{1}{k^{2} \sqrt{\lambda_{2} n}}\left(\frac{\partial k}{\partial \theta}\right) \hat{U}+\frac{1}{k n}\left(\frac{\partial}{\partial \gamma} \log k\right)\left(T_{(1)}-\frac{2}{k}\right)
$$




$$
\begin{aligned}
& -\frac{1}{k^{3} \lambda_{2} n}\left(\frac{\partial k}{\partial \theta}\right)^{2} \hat{U}^{2}+\frac{1}{2 k^{2} \lambda_{2} n}\left(\frac{\partial^{2} k}{\partial \theta^{2}}\right) \hat{U}^{2} \\
& +\frac{\pi_{(1)}}{k^{2} n}+O_{p}\left(\frac{1}{n \sqrt{n}}\right) \\
= & T_{(1)}-\frac{1}{k} \\
& +\frac{1}{k^{2} \sqrt{\lambda_{2} n}}\left(\frac{\partial k}{\partial \theta}\right)\left\{\hat{U}+\frac{1}{\sqrt{\lambda_{2} n}}\left(\frac{1}{k}\left(\frac{\partial \lambda_{1}}{\partial \gamma}\right)+\frac{\lambda_{3}}{2 \lambda_{2}}\right)\right\} \\
& +\frac{1}{k n}\left(\frac{\partial}{\partial \gamma} \log k\right) T_{(1)} \\
& +\frac{1}{2 k^{2} \lambda_{2} n}\left\{\frac{\partial^{2} k}{\partial \theta^{2}}-\frac{2}{k}\left(\frac{\partial k}{\partial \theta}\right)^{2}\right\}\left(\hat{U}^{2}-1\right) \\
& +\frac{B}{k n}+O_{p}\left(\frac{1}{n \sqrt{n}}\right),
\end{aligned}
$$

where

$$
\begin{aligned}
(\mathrm{A} .29) \quad B= & -\frac{1}{k \lambda_{2}}\left(\frac{\partial k}{\partial \theta}\right)\left\{\frac{1}{k}\left(\frac{\partial \lambda_{1}}{\partial \gamma}\right)+\frac{\lambda_{3}}{2 \lambda_{2}}\right\}+\frac{1}{2 k \lambda_{2}}\left\{\frac{\partial^{2} k}{\partial \theta^{2}}-\frac{2}{k}\left(\frac{\partial k}{\partial \theta}\right)^{2}\right\} \\
& -\frac{2}{k}\left(\frac{\partial}{\partial \gamma} \log k\right)+\frac{\pi_{(1)}}{k} .
\end{aligned}
$$

Since, by (3.4)

$$
\frac{\partial \lambda_{1}}{\partial \gamma}=\frac{\partial b_{(1)}}{\partial \theta}=-\frac{\partial k}{\partial \theta},
$$

it follows from (A.29) that

$$
\begin{aligned}
B= & -\frac{1}{2 \lambda_{2}}\left\{\frac{\lambda_{3}}{k \lambda_{2}}\left(\frac{\partial k}{\partial \theta}\right)+\frac{2}{k^{2}}\left(\frac{\partial k}{\partial \theta}\right)\left(\frac{\partial \lambda_{1}}{\partial \gamma}\right)-\frac{1}{k}\left(\frac{\partial^{2} k}{\partial \theta^{2}}\right)+\frac{2}{k^{2}}\left(\frac{\partial k}{\partial \theta}\right)^{2}\right\} \\
& -\frac{1}{k}\left\{2\left(\frac{\partial}{\partial \gamma} \log k\right)-\pi_{(1)}\right\} \\
= & -\frac{1}{2 \lambda_{2}}\left\{\frac{\lambda_{3}}{k \lambda_{2}}\left(\frac{\partial k}{\partial \theta}\right)-\frac{1}{k}\left(\frac{\partial^{2} k}{\partial \theta^{2}}\right)\right\}-\frac{1}{k}\left\{2\left(\frac{\partial}{\partial \gamma} \log k\right)-\pi_{(1)}\right\},
\end{aligned}
$$

which, together with (A.28), yields (4.5). From (4.5) and Theorem A.2 we have

$$
T_{B, \hat{\theta}_{M L}}=T_{(1)}^{* *}+\frac{B}{k n}+O_{p}\left(\frac{1}{n \sqrt{n}}\right),
$$

hence, by (A.3) and (A.4) we obtain (4.6) and (4.7). Thus we complete the proof.

\section{Acknowledgements}

The author thanks the referees for careful reading and valuable comments. Research was supported in part by Grant-in-Aid for Scientific Research (B), No. 23340022 and Grant-in-Aid for Challenging Exploratory Research, No. 15K11992, Japan Society for the Promotion of Science. 


\section{REFERENCES}

Aban, I. B., Meerschaert, M. M. and Panorska, A. K. (2006). Parameter estimation for the truncated Pareto distribution, J. Am. Statist. Assoc., 101, 270-277.

Akahira, M. (1986). The Structure of Asymptotic Deficiency of Estimators, Queen's Papers in Pure and Applied Mathematics 75, Queen's Univ. Press, Kingston, Canada.

Akahira, M. (2016). Second order asymptotic comparison of the MLE and MCLE of a natural parameter for a truncated exponential family of distributions, Ann. Inst. Statist. Math., 68, $469-490$.

Akahira, M., Hashimoto, S., Koike, K. and Ohyauchi, N. (2016). Second order asymptotic comparison of the MLE and MCLE for a two-sided truncated exponential family of distributions, Commun. Statist.-Theory and Meth., 45, 5637-5659.

Akahira, M. and Ohyauchi, N. (2016a). Second order asymptotic loss of the MLE of a truncation parameter for a truncated exponential family of distributions, to appear in Commun. Statist.-Theory and Meth., Published online: 17 June 2016.

Akahira, M. and Ohyauchi, N. (2016b). Second order asymptotic loss of the MLE of a truncation parameter for a two-sided truncated exponential family of distributions, J. Japan Statist. Soc., 46, 27-50.

Akahira, M. and Takeuchi, K. (1982). On asymptotic deficiency of estimators in pooled samples in the presence of nuisance parameters, Statistics \& Decision, 1, 17-38.

Akahira, M. and Takeuchi, K. (1987). On the definition of asymptotic expectation, Foundations of Statistical Inference (eds. I. B. MacNeill and G. J. Umphrey), D. Reidel Publishing Company, 199-208. Also included in: "Joint Statistical Papers of Akahira and Takeuchi", World Scientific, New Jersey, 2003.

Arnold, B. C. (2015). Pareto Distributions, 2nd ed., CRC Press, Boca Raton.

Bar-Lev, S. K. (1984). Large sample properties of the MLE and MCLE for the natural parameter of a truncated exponential family, Ann. Inst. Statist. Math., 36, Part A, 217-222.

Cox, D. R. and Reid, N. (1987). Parameter orthogonality and approximate conditional inference (with discussion), J. Roy. Statist. Soc., Ser. B, 49, 1-39.

Ferguson, H. (1992). Asymptotic properties of a conditional maximum-likelihood estimator, Canad. J. Statist., 20, 63-75.

Hodges, J. L. and Lehmann, E. L. (1970). Deficiency, Ann. Math. Statist., 41, 783-801.

Vancak, V., Goldberg, Y., Bar-Lev, S. K. and Boukai, B. (2015). Continuous statistical models: With or without truncation parameters?, Math. Methods Statist., 24, 55-73. 